\title{
In vitro motility changes in Roux limb after Roux-en-Y reconstruction
}

\author{
Dogan $\mathrm{N}^{1}$, Gokakin AK ${ }^{1}$, Koyuncu A ${ }^{1}$, Bagcivan $\mathrm{I}^{2}$, Aydin $\mathrm{C}^{1}$, Topcu $\mathrm{O}^{1}$, Yildirim $\mathrm{S}^{2}$ \\ Department of General Surgery, School of Medicine, Cumhuriyet University, Sivas, Turkey. \\ dralihan20@hotmail.com
}

\begin{abstract}
Background: The aim of this study is to compare the motility of Roux limb with that of normal segment. Materials and methods: Rats were divided into 3 equal groups as control, Roux-en-Y (Group A) and Roux-en$Y$ with vagotomy (Group B). Only midline incision and manipulations were applied in control group. Following distal gastrectomy, Roux-en-Y reconstruction was applied in Group A, while vagotomy Roux-en-Y gastrojejunostomy was applied in Group B. Rats were sacrificed 1 month later by cervical dislocations under anesthesia. The obtained jejunal segments were cut into four equal parts. The bath was $37^{\circ} \mathrm{C}$ warm while $95 \% \mathrm{O} 2$ and 5 $\%$ CO2 gases were supplied in $10 \mathrm{ml}$ bicarbonate Krebs' solution.

Results: $\mathrm{KCl}$ responses were similar in all three groups. Acetylcholine contraction responses in the vagotomy and non-vagotomy Roux-en-Y groups was higher than in those in control group significantly $(p<0.05)$. This response in vagotomy Roux-en-Y group was also higher than that in non-vagotomy group $(p<0.05)$. The induced electrical field stimulation contraction response in the vagotomy + RouX-en-Y group was lower than those in control group and non-vagotomy group $(p<0.05)$.

Conclusions: These results show that muscarinic receptor density and/or function may increase after vagotomy and non-vagotomy group operation, and vagotomy may contribute to this increase. The decrease in electrical signal response in vagotomy Roux-en-Y group may depend on the decrease in acetylcholine oscillation from the cholinergic nerve ending (Tab. 1, Fig. 5, Ref. 25). Text in PDF www.elis.sk.

Key words: Roux-en-Y, Roux stasis syndrome, acetylcholine, electrical field stimulation, jejunum, motility.
\end{abstract}

Many techniques for restoring the intestinal continuity after gastrectomy have been proposed to compensate for the lost function of the stomach, as well as in attempts to prevent postgastrectomy syndromes (1). Gastrointestinal system continuity can be obtained by Roux-en-Y reconstruction after total or subtotal gastrectomy. After gastric resections, the reconstruction of the gastrointestinal transit in Roux-en-Y should prevent the back flow of the biliary, enteric and pancreatic secretions to the gastric stump. However, some complications may occur during the late postoperative period, as for example, $30 \%$ of the subjects may develop jejunal ulcers near the gastroenteric anastomosis, and mainly, a delayed gastric emptying (2)

Mathias et al (3), described the Roux Stasis Syndrome which is characterized by abdominal pain, nausea, and vomiting worsened after food ingestion. These symptoms may occur due to motor impairment in the Roux-en-Y limb leading to a delayed gastric emptying.

Syndrome occurs partially as a result of intestinal transection needed for Roux-limb formation. Transection separates the Rouxlimb from duodenal pacemaker. As a result, ectopic pacemakers originate 5 and $40 \mathrm{~cm}$ distal to the beginning of the limb. Those

${ }^{1}$ Department of General Surgery, Faculty of Medicine, Cumhuriyet University, Sivas, Turkey, and ${ }^{2}$ Department of Pharmacology, Faculty of Medicine, Cumhuriyet University, Sivas, Turkey

Address for correspondence: A.K. Gokakin, Department of General Surgery, School of Medicine, Cumhuriyet University, 58140, Sivas, Turkey. Phone: +90.346.2580491, Fax: +90.346.2581305 transmit currents that are lower in frequency when compared to duodenal pacemaker. In addition, these pacemakers direct the transetter potentials and as a result, new contractions propagate towards the stomach from Roux-lim. Slow pacesetters and propagation of pacesetter potentials towards mouth delay the gastric evacuation and slow down the transit from roux arm (4-6). One of the mechanisms that facilitate the stasis in the Roux-limb would be the motility disorder. Gastric resection, truncal vagotomy, jejunal transection, and duodenal bypass could be responsible for the occurrence of motility abnormalities by violating the start and regulatory mechanisms of digestive system motor function (7).

Tu et al (8) investigated that Roux stasis syndrome could be improved by performing a corrective "uncut" Roux operation. They found out that in the uncut Roux limb, more pacesetter potentials propagated in the aboral direction than in the standard Roux limb, but gastric emptying was not speeded up by the uncut Roux operation. Additionally, in their experimental study, Zhang et al. demonstrated that uncut Roux-en-Y gastrojejunostomy may lessen the effects of operation on myoelectric activity such as slow waves, spike potential, as well as decrease in the impairment of gastrointestinal motility (9).

Le Blanc-Louvry et al (10) demonstrated that distal or total gastrectomy with a Roux-en-Y limb induce marked disturbances in normal jejunal motor patterns during the fasting and non-fasting states This emphasizes the possible central role of a very slow migration velocity in postoperative symptoms. 
Although studies mentioned above have tried to clarify the mechanisms of Roux stasis by pacemaker change and retrograde motility, they cannot explain the exact reason of Roux stasis syndrome. It was our aim in this study to investigate the in vitro response of small intestine smooth muscle in the Roux limb to parasympathetic drugs and electrical field stimulation.

\section{Material and methods}

The investigation was conducted in accordance with the Guide for the Care and Use of Laboratory Animals published by the US National Institutes of Health (NIH Publication no. 85-23, revised 1996) and the approval has been received from Institutional Animal Ethics Committee at Cumhuriyet University (06/24/2011-927).

\section{Animals}

A total of 30 adult Wistar Albino rats weighing 225 to $350 \mathrm{~g}$ were included in the study. Animals were provided by the Experimental Animals Center, Cumhuriyet University, Sivas, Turkey. The animals were fed ad libitum with standard diet and water throughout the experiment. All animals were housed separately and kept under standard conditions of room temperature $\left(22-24^{\circ} \mathrm{C}\right)$ and a 12 h light/12 h dark cycle.

\section{Exerimental design}

Isofluran (Abbott) inhibition and intraperitoneal sodium pentothal (Ampro) $(50 \mathrm{mg} / \mathrm{kg}$ ) were used for anesthesia in all rats before surgical procedures. Median incision of $2 \mathrm{~cm}$ in length was used for laparotomy. Duodenum, jejunum, and stomach were only manipulated, and abdomen was closed by sutures after one hour in control group. In Group A, Roux en Y gastrojejunostomy was performed after distal gastrectomy. In the third group (Group B), distal gastrectomy and Roux-en-Y gastrojejunostomy and bilateral truncal vagotomy was done.

Rats were sacrificed after one month by cervical dislocation, and then laparotomy was performed. Roux-limbs from Roux-en-Y groups and reciprocal jejunal segments were resected in control group. These resected materials were put into Krebs solution and transferred to pharmacologic laboratory immediately. Jejunal segments were cleaned from surrounding tissues and cut into 4 strips in width and $1 \mathrm{~cm}$ in length. The solution was gassed with $95 \%$ $\mathrm{O}_{2}$ and $5 \% \mathrm{CO}_{2}$ during the study, and its temperature was maintained at $37^{\circ} \mathrm{C}$ by means of a thermo-regulated water circuit. One end of each preparation was attached to the bottom of the organ bath while the other end was tied to a force transducer (Grass FT 03) connected to a pen polygraph (Grass $79 \mathrm{E}$ ). After mounting, each strip was allowed to equilibrate with basal tension of $1.5 \mathrm{~g}$ for 15 minutes, and during this time, Krebs solution was replaced every 15 min with fresh solution. Jejunal strips were contracted with $80 \mathrm{mM} \mathrm{KCL}$ after equilibration. KCL application was done to prevent possible spontaneous contractions during experiment and to evaluate contraction results. Afterwards, the washed tissues were allowed to relax for 30 minutes in order to get ready for being treated with substances.
Jejunum tissues obtained from control and experiment groups had been treated with $80 \mathrm{mM} \mathrm{KCL}$ before antagonist drugs were given. Contractile responses elicited by KCL were demonstrated as mg in graphs.

\section{Acetylcholine contractile responses}

Jejunum strips from all three groups were treated with addition of acetylcholine $\left(10^{-9}-10^{-4} \mathrm{M}\right)$ in cumulative doses, and concentration-response curves were obtained. After the contractile response had reached a steady state at any concentration, a higher concentration was applied. The contractions produced by acetylcholine in study and control groups were graphed as percentage of contraction elicited by KCL.

\section{Contractile responses produced by electrical field stimulations}

Ten-second EFS of $50 \mathrm{~V}, 0.8 \mathrm{msn}, 2-64 \mathrm{~Hz}$ frequency was given to jejunal strips obtained from control and study groups. The produced contractions were graphed as percentage of contraction elicited by KCL.

Drugs and solutions used in study

- Krebs bicarbonate solution in mM/L: $\mathrm{NaCl} 118 ; \mathrm{KCl} 4.7 ; \mathrm{CaCl}_{2}$ 2.5; $\mathrm{NaHCO}_{3} 25 ; \mathrm{MgSO}_{4}$ 1.2; $\mathrm{KH}_{2} \mathrm{PO}_{4}$ 1.2; Glucose.

- Isoflurane (Abbott laboratories, Norfolk, Chicago)

- Pentobarbital (Ampro Pharmacy, Arcadia CA), Acetylcoline (Sigma)

All drugs were dissolved in water and prepared daily for every experiment.

\section{Statistical evaluation}

In order to evaluate the effects of agonists; maximum responses (Emax) and $\mathrm{pD}_{2}$ values (apparent agonist affinity constants; -Log ED50) were calculated. The concentration-response data obtained in each individual experiment were plotted as response/concentration (Y), agonist response (X). This produced a straight-line relationship in each experiment as predicted from the standard equation for drug receptor interaction. Data were expressed as mean \pm standard deviation. Statistical comparison between groups were performed using general linear models of analysis of variance (ANOVA) followed by Scheffe's F test and p values of less than 0.05 were considered to be statistically significant.

$$
\begin{aligned}
& \mathrm{pD}_{2}=\log \mathrm{A}-\log \left(\mathrm{E}_{\max } / \mathrm{E}_{\mathrm{A}}-1\right) \\
& \mathrm{A}=\text { Molar concentration of agonist drugs } \\
& \mathrm{E}_{\max }=\text { Maximum effect of agonist drugs } \\
& \mathrm{E}_{\mathrm{A}}=\text { Effect of certain concentration of agonist drugs }
\end{aligned}
$$

\section{Results}

\section{KCL contractile responses}

Contractile response that was induced with $80 \mathrm{mM}$ KCL produced no significant difference in study and control groups (Fig. 1).

\section{Acetylcholine contractile responses}

Acetylcholine $\left(10^{-9}-10^{-4} \mathrm{M}\right)$ produced contractions in a concentration-dependent manner in isolated strips in all three groups. 


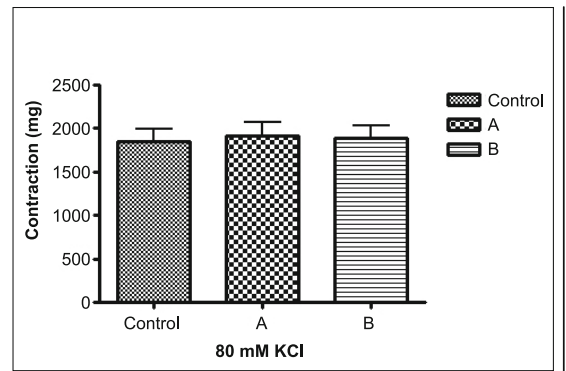

Fig. 1. Contractile response with $80 \mathrm{mM}$ KCL.



Fig. 2. Acetylcholine contractile responses. The values of $\mathrm{pD}_{2}$.

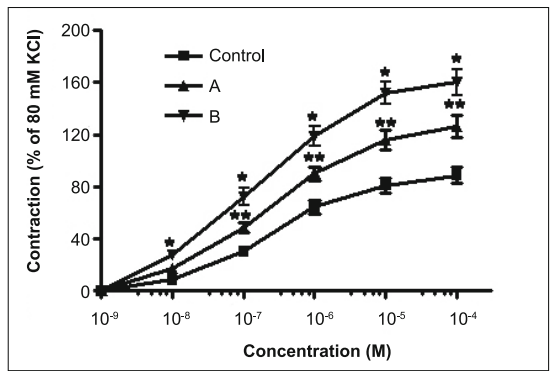

Fig. 3. Acetylcholine contractile responses. The values of $\mathrm{pD}_{2}$.



Fig. 4. EFS-induced contractions.

Fig. 5. EFS-induced contractions.

Acetylcholine-induced contractions were increased significantly in Group A (Roux-en-Y) compared to control group ( $\mathrm{p}<0.05$ ). These contractions were also increased significantly in vagotomized Roux-en-Y group (Group B) compared to non-vagotomized Roux-en-Y group (Group A) ( $<<0.05)$. The values of $\mathrm{pD}_{2}$ were similar in all three groups (Figs 2 and 3, Tab. 1).

Contractile responses induced by electrical field stimulation (EFS)

Frequency-dependent $(2-64 \mathrm{~Hz})$ contractions were obtained from isolated jejunal strips in all three groups. EFS-induced contractions were decreased significantly in Group B (vagotomy+Roux en $\mathrm{Y}$ ) compared to control and Group A (Roux-en-Y) $(\mathrm{p}<0.05)$ (Figs 4 and 5; Tab. 1).

\section{Discussion}

The advantages of R-Y reconstruction are known as prevention of reflux esophagitis, gastritis and gastric cancer in the residual stomach, as well as reduction in risk of suture rupture (11) and

Tab. 1. Emax and $\mathrm{pD}_{2}$ values in isolated jejunum strips of rat.

\begin{tabular}{lccc}
\hline & Control & Group A & Group B \\
\hline KCl & & & \\
$\quad$ Emax(mg) & $1848 \pm 144$ & $1920 \pm 156$ & $1892 \pm 145$ \\
\hline Acetylcholine & & & \\
$\quad$ Emax(\%) & $88,7 \pm 6,4$ & $126,6 \pm 8,6 *$ & $160,3 \pm 10,1^{* *}$ \\
$\quad$ PD2 & $6,86 \pm 0,07$ & $6,93 \pm 0,07$ & $6,99 \pm 0,10$ \\
\hline EAU & & & \\
$\quad$ Emax(\%) & $91,2 \pm 8,8$ & $82,3 \pm 7,8$ & $60,1 \pm 6,0 * *$ \\
\hline
\end{tabular}

* Statistically significant as compared the control group.

** Statistically significant as compared control and Group A (Roux en Y) tendency to vomit via preventing the duodenal content from entering the stomach following gastroenterostomy (12). It is performed mostly to divert the pancreaticobilary juice and thus to prevent reflux gastritis or esophagitis after Billroth I and II operations $(3,13)$.

The symptoms of gastric retention, such as abdominal pain, nausea, vomiting, abdominal distention, and loss of appetite occur in approximately in $30 \%$ of patients who undergo an R-Y after distal gastrectomy $(4,11)$. This syndrome, called the Roux stasis syndrome, is caused in part by motility disorder of the Roux limb $(4,14)$. Two factors that may contribute to the cause of syndrome are the disruption of propagation of pacesetter potentials from duodenal pacemaker to Roux limb, and appearance of ectopic pacemaker in the limb $(11,15,16)$.

It was reported that gastric emptying was delayed in dogs that had undergone gastrectomy and Roux-en-Y gastroenterostomy. Quantitative and qualitative changes in Roux limb motility were documented. Roux dogs emptied their stomachs after solid meal four times more slowly than control animals (17). Previously, these investigators also reported that Roux animals emptied more slowly than comparable dogs with Billroth II gastrojejunostomy, and indicated that the reason for the stasis lied in Roux limb motility changes (18). Hinder et al (19) have reported that the reason for the Roux stasis syndrome lied in the gastric remnant, while extensive gastric resection is an effective means to reduce symptoms and improve gastric emptying in selected patients with severe gastric stasis of solid food after the Roux-en-Y procedure. As opposed to latter authors, Dutre et al (2) showed that besides being unable to protect the stomach from the enterogastric reflux, the standard Roux limb may become a functional barrier for gastric emptying. These results suggested that jejunal transection may be an important factor for Roux en Y syndrome. 
Tu et al (15) reported that un-cut Roux operation eliminated the Roux stasis syndrome by preserving neuromuscular continuity between the proximal jejunum and Roux limb. Mon et al (20) showed that Visick grade improved following the uncut Roux procedure, but changed little after standard Roux reconstruction, when comparing clinical results in patients who have undergone uncut Roux-en-Y gastrojejunostomy with those in patients who have undergone the standard Roux-en-Y gastrojejunostomy. Additionally, in an experimental study in pigs, Kiciak et al (21) demonstrated that the 'uncut' Roux group returned to their normal physical movement, felt hungry and looked for food earlier than Roux-en-Y group.

It was showed that enterogastric reflux occurred in dogs with vagotomy and Roux gastrectomy. Ectopic pacemaker in Roux limb which drove the pacesetter potentials of the limb in a reverse or oral direction was also showed. It was discussed that the ectopic pacemakers contributed to, but were not solely responsible for enterogastric reflux after operation. The appearance of the ectopic pacemaker in the Roux limb and the decrease in frequency of the pacesetter potential in Roux limb were likely caused by jejunal transection required with the Roux operation and did not result from vagotomy or gastrectomy (22).

The role of vagal denervation in the etiology of this "Rouxstasis syndrome” is controversial. Animals with gastric vagotomy had a significantly faster rate of gastric emptying than those with truncal vagotomy. Therefore the extragastric vagal innervation appears to play a role in determining the rate of emptying solids after antrectomy and Roux-en-Y gastrojejunostomy (23). In a canine model, selective vagotomy does not prevent delayed gastric emptying or myoelectric alterations following Roux-en-Y (24).

The etiology of delayed gastric emptying and myoelectrical changes has not been cleared yet. The possible causes include distruption of myoneuronal continuity of intestine due to separation of roux arm from duodenal pacemaker (1); vagal denervation of stomach and/or small intestine (2); duodenal bypass with changed secretion of gastrointestinal regulatory hormones (3); decreased liquefaction of nutriments in consequence of the diversion of pancreatic and biliary secretions from gastric remnant (4) and/or malabsorbtion-induced reflex decrease in gastric emptying mediated by neuroal and/or humoral pathways in the presence of unabsorbed carbohydrates and/or fats in the ileum (5) (24).

All those studies mentioned above lead us to think that some changes in contractility response may have occurred in the smooth muscle of Roux limb. In this in vitro study, the contractility of Roux limb jejunum smooth muscle was compared to that of normal jejunum smooth muscle. In addition the effect of vagotomy was evaluated.

We demonstrated that contractility responses to $80 \mathrm{mM} \mathrm{KCl}$ in experiment group were not different significantly from that in control group, which indicates that the contractile function of smooth muscle changed neither in control nor in experiment groups.

Frequency-dependent EFS induced contractile responses obtained in isolated jejunal strips in all three groups. When control and experiment groups were compared, EFS-induced contractions in Roux-en-Y + vagotomy were decreased significantly compared to Roux-en-Y only and control groups. These results suggest a decrease in acetylcholine secretion from cholinergic nerve terminals in Roux-en-Y + vagotomy group in contrast to Roux-en-Y only, and control groups, where no change may have occurred.

In all three groups, acetylcholine produced concentrationdependent contractions in isolated jejunal strips. Contractile responses increased significantly in roux-en-Y groups compared to control group. The values of $\mathrm{pD}_{2}$ were similar in all three groups. These results suggest that an increase in muscarinic reseptor density and/or function may have occurred after roux-en-Y reconstruction independent of vagotomy. On the other hand, higher rate of contractions in roux-en-Y + vagotomy group compared to Rouxen-Y only group suggest an even greater increase in muscarinic function brought on by the effect of vagus cut. Similar $\mathrm{pD}_{2}$ values in all three groups may suggest that no change has occurred in the affinity of muscarinic agonists to receptors.

In a study that has been done on nicotinic and muscarinic acethylcholine receptors in rats it was shown that surgical vagal denervation increased the density of acethylcholine receptors located in the parasympathetic ganglion cells. As nicotinic acethylcholine receptors increase after subdiaphragmatic vagotomy, the remaining of muscarinic acethylcholine receptors was explained by degeneration preganglionic neurons in stomach after vagotomy (25).

\section{Conclusion}

Consequently, further studies focused e.g. on receptor determination, receptor levels, post-receptor events (intracellular macromolecules, second messengers, intracellular ion levels) are needed in order to determine the exact cause of the changes that occurred in contraction responses after Roux-en-Y + vagotomy and Roux-en-Y only operations.

\section{References}

1. Shibata C, Ueno T, Kakyou M, Kinouchi M, Sasaki I. Results of reconstruction with jejunal pouch after gastrectomy: Correlation with gastrointestinal motor activity. Dig Surg 2009; 26: 177-186.

2. Dutra RA, Araujo WM, Andrade JI. The effects of roux-en-y limb length on gastric emptying and enterogastric reflux in rats. Acta Cir Bras 2008; 23: 179-183.

3. Mathias JR, Fernandez A, Sninsky CA, Clench MH, Davis RH. Nausea, vomiting, and abdominal pain after roux-en-y anastomosis: Motility of the jejunal limb. Gastroenterology 1985; 88: 101-107.

4. Tu BN, Kelly KA. Motility disorders after roux-en-y gastrojejunostomy. Obes Surg 1994; 4: 219-226.

5. Morrison P, Miedema BW, Kohler L, Kelly KA. Electrical dysrhythmias in the roux jejunal limb: Cause and treatment. Am J Surg 1990; 160: 252-256.

6. Cullen JJ, Eagon JC, Hould FS, Hanson RB, Kelly KA. Ectopic jejunal pacemakers after jejunal transection and their relationship to transit. Am J Physiol 1995; 268: G959-967.

7. Schumpelick V AG (Ed). Surgical intervention, Hampshire Wrightson Biomedical, 1990, 167-182. 


\section{0-344}

8. Tu BL, Kelly KA. Surgical treatment of roux stasis syndrome. J Gastrointest Surg 1999; 3: 613-617.

9. Zhang YM, Liu XL, Xue DB, Wei YW, Yun XG. Myoelectric activity and motility of the roux limb after cut or uncut roux-en-y gastrojejunostomy. World J Gastroenterol 2006; 12: 7699-7704.

10. Le Blanc-Louvry I, Ducrotte P, Peillon C, Michel P, Chiron A, Denis P. Roux-en-y limb motility after total or distal gastrectomy in symptomatic and asymptomatic patients. J Am Coll Surg 2000; 190: 408-417.

11. Hoya Y, Mitsumori N, Yanaga K. The advantages and disadvantages of a roux-en-y reconstruction after a distal gastrectomy for gastric cancer. Surg Today 2009; 39: 647-651.

12. Kirk RM. Roux-en-y. World J Surg 1985; 9: 938-944.

13. Madura JA. Primary bile reflux gastritis: Which treatment is better, roux-en-y or biliary diversion? Am Surg 2000; 66: 417-423; discussion 423-414.

14. Masui T, Kubora T, Nakanishi Y, Aoki K, Sugimoto S, Takamura M, Takeda H, Hashimoto K, Tokuka A. The flow angle beneath the gastrojejunostomy predicts delayed gastric emptying in roux-en-y reconstruction after distal gastrectomy. Gastric Cancer 2012; 15: 281-286.

15. Tu BN, Kelly KA. Elimination of the roux stasis syndrome using a new type of “uncut roux" limb. Am J Surg 1995; 170: 381-386.

16. Hirao M, Fujitani K, Tsujinaka T. Delayed gastric emptying after distal gastrectomy for gastric cancer. Hepatogastroenterology 2005; 52: 305-309.

17. Harrison WD, Hocking MP, Vogel SB. Gastric emptying and myoelectric activity following roux-en-y gastrojejunostomy. J Surg Res 1990; 49: 385-389.
18. Hocking MP, Vogel SB, Falasca CA, Woodward ER. Delayed gastric emptying of liquids and solids following roux-en-y biliary diversion. Ann Surg 1981; 194: 494-501.

19. Hinder RA, Esser J, DeMeester TR. Management of gastric emptying disorders following the roux-en-y procedure. Surgery 1988; 104: 765-772.

20. Mon RA, Cullen JJ. Standard roux-en-y gastrojejunostomy vs. "Uncut” roux-en-y gastrojejunostomy: A matched cohort study. J Gastrointest Surg 2000; 4: 298-303.

21. Kiciak A, Wolinski J, Borycka K, Zabielski R, Bielecki K. Rouxen-y or 'uncut' roux procedure? Relation of intestinal migrating motor complex recovery to the preservation of the network of interstitial cells of cajal in pigs. Exp Physiol 2007; 92: 399-408.

22. Karlstrom L, Kelly KA. Ectopic jejunal pacemakers and gastric emptying after roux gastrectomy: Effect of intestinal pacing. Surgery 1989; 106: 867-871.

23. Woodward A, Schu W, Wojtowycz AR, Sillin LF. Gastric emptying of solids after roux-en-y gastrectomy: Is extragastric vagal innervation important? Surgery 1991; 110: 793-797; discussion 797-798.

24. Hocking MP, Carlson RG, Vogel SB. Does selective vagotomy prevent delayed gastric emptying and altered myoelectric activity following roux-en-y gastrojejunostomy? Am J Surg 1992; 163: 32-35; discussion 35-36.

25. Nishikawa H, Taniguchi T, Ninomiya H, Fujiwara M. Nicotinic acetylcholine receptors in the rat stomach: Ii. Alterations after vagotomy. Eur J Pharmacol 1988; 146: 105-111.

Received October 11, 2012. Accepted March 8, 2014. 